This is the accepted version of the article:

Bhaskar U.K., Banerjee N., Abdollahi A., Solanas E., Rijnders G., Catalan G.. Flexoelectric MEMS: Towards an electromechanical strain diode. Nanoscale, (2016). 8. : 1293 . 10.1039/c5nr06514c.

Available at: https://dx.doi.org/10.1039/c5nr06514c 


\title{
Flexoelectric MEMS: towards an electromechanical strain diode $\dagger$
}

\author{
U. K. Bhaskar, ${ }^{*} \ddagger^{a}$ N. Banerjee,$\ddagger^{b}$ A. Abdollahi, ${ }^{a}$ E. Solanas, ${ }^{d}$ G. Rijnders ${ }^{b}$ and \\ G. Catalan ${ }^{a, c}$ \\ DOI: $10.1039 / \mathrm{c} 5 \mathrm{nr} 06514 \mathrm{c}$
}

\begin{abstract}
Piezoelectricity and flexoelectricity are two independent but not incompatible forms of electromechanical response exhibited by nanoscale ferroelectrics. Here, we show that flexoelectricity can either enhance or suppress the piezoelectric response of the cantilever depending on the ferroelectric polarity and lead to a diodelike asymmetric (two-state) electromechanical response.
\end{abstract}

Piezoelectricity allows non-centrosymmetric crystals to efficiently transduce electrical energy into mechanical energy and vice versa. In most microelectromechanical systems (MEMSs), ${ }^{1,2}$ piezoelectric materials are used together with a non-piezoelectric elastic layer in a composite structure, referred to herein as a bimorph structure. The mechanical clamping induced by the nonpiezoelectric layer helps translate the homogeneous piezoelectric strains generated on application of an electrical bias (converse piezoelectricity) into a flexural motion: the region of the piezoelectric closest to the interface with the inert material deforms less than the region farthest away, thereby creating a deformation gradient and hence a bending.

Meanwhile, converse flexoelectricity ${ }^{3-9}$ is a different electromechanical coupling that also allows any insulating material, irrespective of its symmetry, to bend in response to an applied voltage. Flexoelectric bending arises from deformation at the unit cell level ${ }^{6}$ and does not require any clamping layer. Flexoelectricity is not inherently incompatible with piezoelectricity, so in piezoelectric bimorph actuators flexoelectric bending must exist in addition to the piezoelectric bending. However, flexoelectricity, when compared to piezoelectricity, is a weak effect of little practical significance in bulk materials, so its con-

\footnotetext{
${ }^{a}$ ICN2 Institut Catala de Nanociencia $i$ Nanotecnologia, CSIC and The Barcelona Institute of Science and Technology (BIST), Campus UAB, 08193 Bellaterra, Barcelona, Spain. E mail: gustau.catalan@icn.cat, umeshkbhaskar@gmail.com ${ }^{b}$ Faculty of Science and Technology and MESA+ Institute for Nanotechnology, University of Twente, P.O. Box 217, 7500 AE Enschede, The Netherlands

'ICREA Institucio Catalana de Recerca $i$ Estudis Avançats, 08010 Barcelona, Spain

${ }^{d}$ Lyncée Tec SA, PSE A, CH 1015 Lausanne, Switzerland

$\dagger$ Electronic supplementary information (ESI) available. See DOI: 10.1039/ c5nr06514c

\Both authors contributed equally.
}

tribution to the actuator performance has so far gone unnoticed. In MEMS, however, the balance of power between piezoelectricity and flexoelectricity is shifted, because any (strain) gradient scales inversely with the thickness, and MEMS can be very thin, so flexoelectricity can become large.

It has been theoretically predicted ${ }^{10,11}$ that the interaction between flexoelectricity and piezoelectricity in nanocantilevers can lead to a significant size-dependent enhancement of the effective (piezoelectric + flexoelectric) coefficients, a prediction that still awaits experimental verification. There is however an additional consideration when the piezoelectric layer is ferroelectric: the sign of the piezoelectric coefficient (a third rank tensor) can be switched by applying a sufficiently large (coercive) voltage, whereas the sign of flexoelectricity (a fourth rank tensor) is independent of the ferroelectric polarity. Therefore, two distinct regimes of operation should in theory exist: when flexoelectricity and ferroelectricity align opposite to each other, it results in a decreased or eventually even cancelled deformation, and when they align together, it results in an enhanced deformation (Fig. 1(d)). The asymmetry of this interaction has been demonstrated in bulk pyramidal ceramic lead magnesium niobate-lead titanate. ${ }^{12}$ At the nanoscale, this asymmetry offers the possibility of engineering a new type of electromechanical device with inherent two-state functionality, namely one where the magnitude of mechanical deformation depends on the ferroelectric polarity. In this paper, by using a $\mathrm{Pb}\left(\mathrm{Zr}_{0.52}, \mathrm{Ti}_{0.48}\right) \mathrm{O}_{3}$ (PZT)-based ferroelectric bimorph MEMS cantilever, we demonstrate that piezoelectric and flexoelectric effects can indeed be combined to achieve such a two-state functionality in ferroelectric MEMS yielding a deformation amplitude that depends on the sign of the applied voltage.

In this work, all-oxide nanocantilevers (Fig. 1(a and b)) were fabricated as capacitor structures with a PZT active layer sandwiched between two $\mathrm{SrRuO}_{3}$ (SRO) electrodes. The whole structure was epitaxially grown on a buffer of Yttria-stabilized Zirconia (YSZ) on Si. The fabrication method utilized the liftoff patterning ${ }^{13}$ of the epitaxial oxide stack, followed by release of the freestanding devices using anisotropic etching. In contrast to the traditional top-down patterning, the lift-off process 
(a)

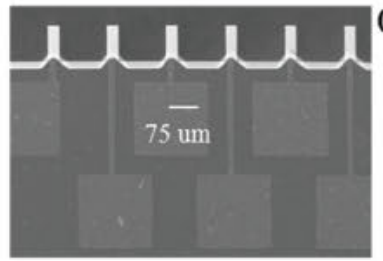

(d) (b)

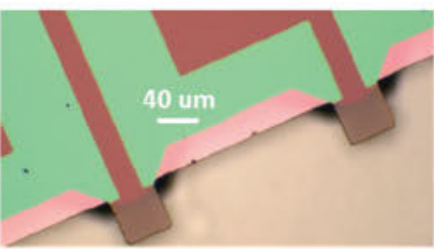

MFlexo+MPiezo (c)

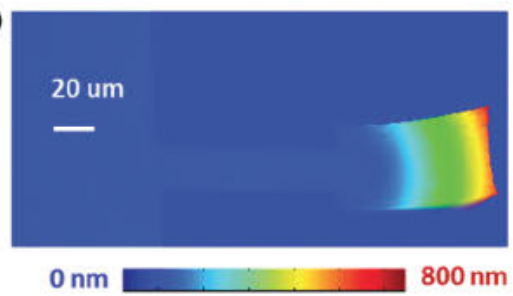

$0 \mathrm{~nm}$

$800 \mathrm{~nm}$
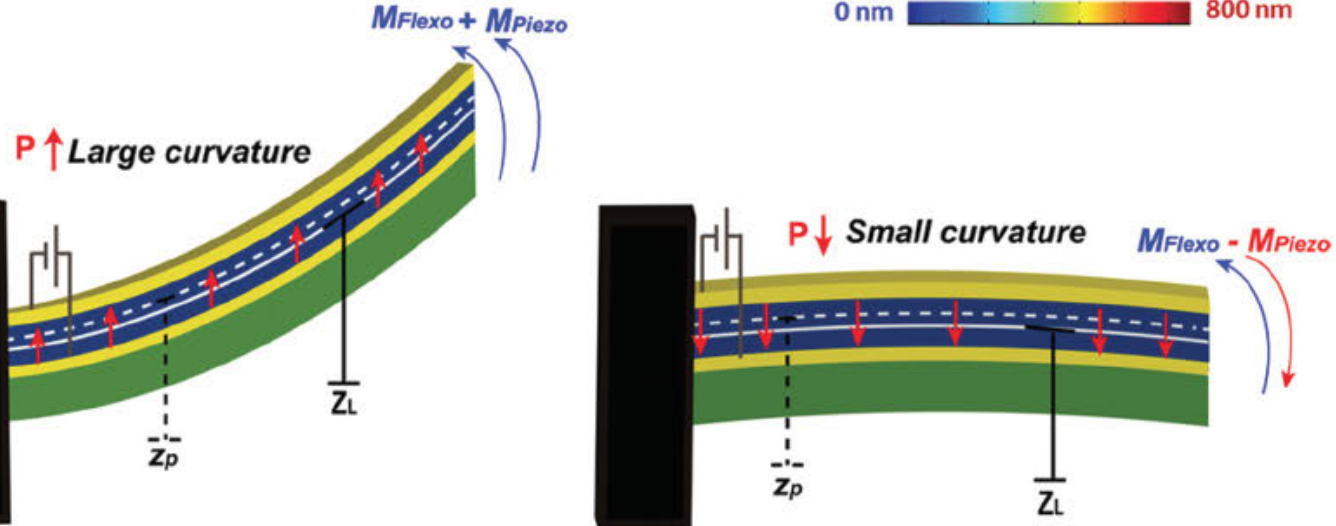

- Ferroelectric layer PZT = Electrode SRO = Elastic layer YSZ

Fig. 1 (a) Scanning electron microscopy and (b) optical image of an array of PZT nanocantilevers; (c) 3D image of one PZT nanocantilever with colour scale corresponding to the out of plane displacement; (d) schematic illustration of piezoelectric and flexoelectric moments working together for ferroelectric polarisation in the negative $z$ axis and cancelling each other for ferroelectric polarisation in the positive $z$ axis.

employs a sacrificial layer of amorphous $\mathrm{AlO}_{x}$ mask and structures the hetero-epitaxial multilayer in a single lift-off step. All the oxide layers were grown by pulsed laser deposition. The buffer layer of YSZ was utilized to promote the epitaxial growth of the perovskite layers on $\mathrm{Si}$, and it also acted as the elastic layer in the bimorph actuation in the final devices. Following the growth and lift-off patterning of the heterostructures, the top $\mathrm{SrRuO}_{3}$ electrode layer was defined using ion-beam etching. The PZT films are oriented with the perovskite $\{110\}$ planes parallel to the surface. The dielectric and ferroelectric properties of the PZT films grown under similar conditions are reported in ref. 13-15. At the frequency of measurement $(1.3 \mathrm{kHz})$, the dielectric loss $\tan (\delta)$ is $<0.1$, indicating that the released cantilevers are dielectrically sound and not leaky. The final free-standing devices were released by anisotropic etching of the $\mathrm{Si}(100)$ substrate employing a basic solution. ${ }^{16}$

In order to facilitate inference by comparison, piezoelectric bimorph cantilevers of two thicknesses of PZT - 150 and $75 \mathrm{~nm}$ were fabricated. In both cases, the thickness of the YSZ elastic layer was the same as that of the piezoelectric layer, while the thickness of the top and bottom SRO electrode was $25 \mathrm{~nm}$. Finally, a "asymmetry amplifier" was made from a $100 \mathrm{~nm}$ thick PZT bimorph structure having a thick SRO electrode $\sim 100 \mathrm{~nm}$ for the top electrode. The thicker top electrode partially compensates for the non-centrosymmetry introduced by the $100 \mathrm{~nm}$ thick YSZ layer and the $25 \mathrm{~nm}$ thick SRO bottom electrode, thus increasing the relative strength of flexoelectric vs. piezoelectric bending and therefore also the asymmetry. Using an analytical model that we describe in this article, we have created an application that calculates the asymmetry in bending as a function of polarity for any user-specified material coefficients and geometric parameters, so as to allow potential users to design ferroelectric MEMS devices with a desired level of electromechanical asymmetry (https://umeshkbhaskar.shinyapps.io/pzt app/). The calculations show that, for the right parameter combination, it is theoretically possible to achieve a diode-like behaviour whereby the cantilever bending is more than an order of magnitude bigger for one polarity than for the opposite.

The observation of out-of-plane oscillations (Fig. 1(c)) induced by applying an electrical excitation to the released cantilevers was made using a commercial (lyncee tec) Digital Holographic Microscope (DHM) ${ }^{17,18}$ working in stroboscopic mode. The displacement $(z)$ was measured at multiple points along the length of the cantilever (ESI Fig. $1 \dagger$ ), and the curvature $(\kappa)$ was calculated as $\partial^{2} z / \partial x^{2}$, where $x$ is the horizontal distance to the clamping point.

The curvature of the cantilever, along with the applied sinusoidal excitation, is plotted in Fig. 2 as a function of time, both for the case when the polarization vector is pointing in the positive $z$ axis, referred to as $P_{+}$(colored blue in Fig. 2(a)), and the opposite case, referred to as $P_{-}$(colored red in Fig. 2 (b)). The direction of voltage sweep for both $P_{+}$and $P_{-}$is unipolar, and is chosen so as to favor the existing polarization; for example, a sinusoidal excitation spanning only negative voltages is applied to favor $P_{+}$. This is done in order to avoid additional electromechanical contributions from switching during voltage application. The curvature as a function of applied electric field for the full bipolar voltage cycle is also shown in Fig. 3 (a) for the $150 \mathrm{~nm}$, and (b) $75 \mathrm{~nm}$ thick PZT bimorph, showing the characteristic butterfly-like hysteresis 

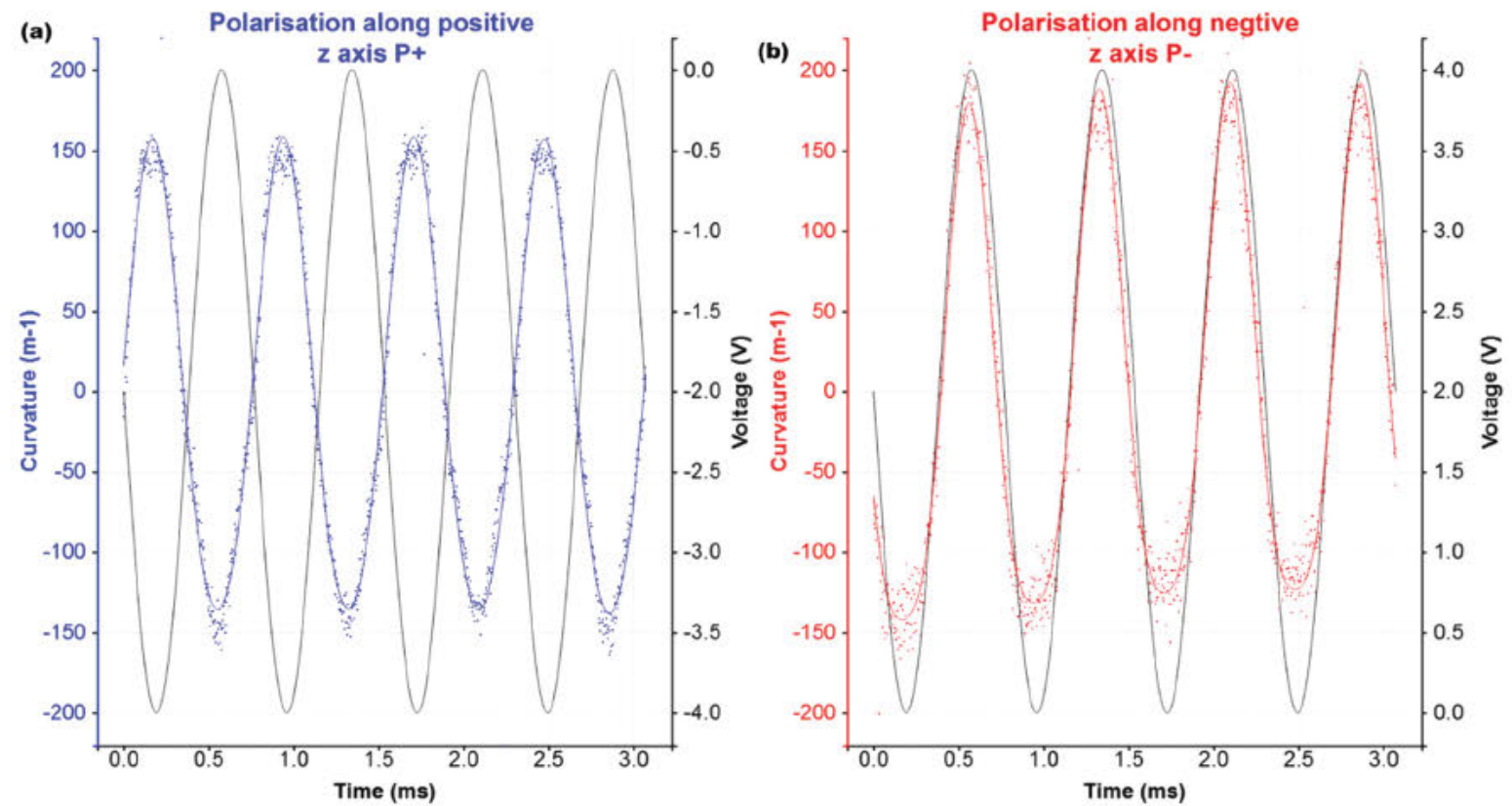

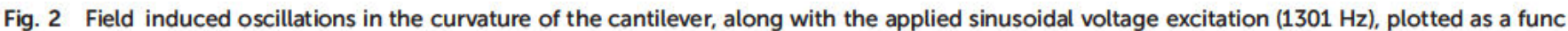

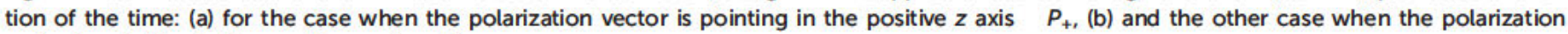
vector is pointing in the negative $z$ axis $P$.
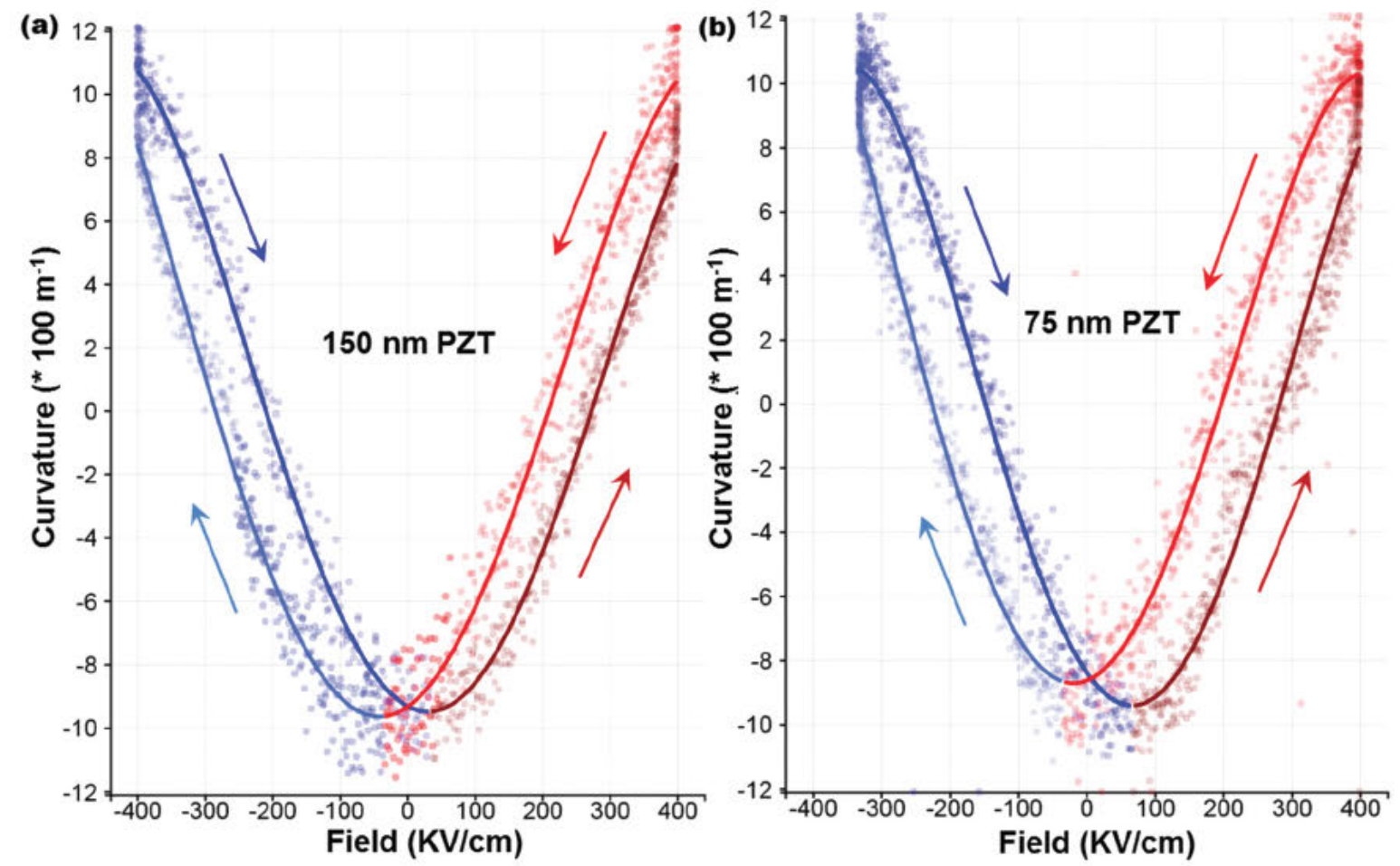

Fig. 3 Butterfly hysteresis loops of curvature as a function of electric field for (a) $150 \mathrm{~nm}$ thick and (b) $75 \mathrm{~nm}$ thick PZT bimorph.

loops expected from the switching piezoresponse of a ferroelectric cantilever. ${ }^{19}$

For an up-polarized sample $P_{+}$, applying a negative voltage at the top electrode increases the ferroelectric polarization, causing an out-of-plane expansion and in-plane contraction of the ferroelectric layer that results in an upward bend of the cantilever (Fig. 1(d)). Conversely, if the ferroelectric polarization is pointing down $P_{-}$, a negative voltage will cause the 
polarization to decrease in magnitude, thus contracting the ferroelectric layer out-of-plane and expanding it in-plane, which results in a downward bend. Meanwhile, the flexoelectrically-induced bending depends only on the sign of the voltage, with a negative voltage resulting in a upward flexoelectric bend because the flexoelectric coefficient for 110oriented perovskites is negative. ${ }^{20}$ Thus, for $P_{+}$polarity, flexoelectricity and piezoelectricity both try to bend the cantilever upwards as a function of increasing voltage, whereas for

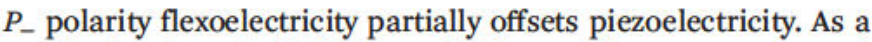
result, the slope $\partial \kappa / \partial V$ (where $\kappa$ is the cantilever curvature and $\mathrm{V}$ is the applied voltage) must be bigger for $P_{+}$than for $P_{-}$.

We can calculate this effect analytically. The first step is to determine the $z$ position of the torque neutral axis $\left(z_{\mathrm{m}}\right)$, a concept well known in engineering mechanics, ${ }^{21,22}$ which is defined as the weighted centre $\left(z_{i}\right)$ of the product of Young's modulus $\left(E_{i}\right)$ and cross-sectional area $\left(A_{i}\right)$ for all $i$ layers in the bimorph structure:

$$
z_{\mathrm{m}}=\frac{\sum_{i} z_{i} E_{i} A_{i}}{\sum_{i} E_{i} A_{i}}
$$

$\frac{\partial \kappa}{\partial V}$ can now be expressed as: ${ }^{23}$

$$
\frac{\partial \kappa}{\partial V}=\frac{E_{\mathrm{p}} Z_{\mathrm{p}} d_{31}}{\sum_{i} E_{i} t_{i}\left(\frac{t_{i}^{2}}{12}+Z_{i}^{2}\right)},
$$

where $E_{\mathrm{p}}$ is the Young's modulus of the PZT layer, $t_{i}$ is the thickness of the layer $i, Z_{i}$ is the position of the centre of layer $i$ with respect to the neutral axis, and $d_{31}$ is the intrinsic piezoelectric coefficient. Assuming a flexoelectric coefficient $\mu_{12}$, the numerator in eqn (2) can be explicitly expanded as $E_{\mathrm{p}} Z_{\mathrm{p}} d_{31}+$ $\mu_{12}$ for $P_{+}$, and $E_{\mathrm{p}} Z_{\mathrm{p}} d_{31}-\mu_{12}$ for $P_{-}$. Thus, $\frac{\partial \kappa}{\partial V}$ can now be expressed as

$$
\frac{\partial \kappa}{\partial V}=\frac{E_{\mathrm{p}} Z_{\mathrm{p}} d_{31}^{\text {eff }}}{\sum_{i} E_{i} t_{i}\left(\frac{t_{i}^{2}}{12}+Z_{i}^{2}\right)},
$$

where

$$
d_{31}^{\text {eff }}=d_{31} \pm \frac{\mu_{12}}{E_{\mathrm{p}} Z_{\mathrm{p}}}
$$

We see then that the combination of piezoelectricity $\left(d_{31}\right)$ and flexoelectricity $\left(\mu_{12}\right)$ yields a polarity-dependent value of the effective piezoelectricity $\left(d_{31}^{\text {eff }}\right)$. This asymmetry is sizedependent, because the flexoelectric contribution (second term on the right side of eqn (4)) scales in inverse proportion to $Z_{\mathrm{p}}$. Though we have calculated it analytically for cantilevers, the existence and size dependence of piezoelectric asymmetry is a more general principle that applies even to bulk piezoelectric devices. ${ }^{12}$ In Fig. 4(a), we plot the curvature as a function of voltage for the $150 \mathrm{~nm}$ thick PZT bimorph structure, using only the data from the reverse sweeps of $P_{+}$and $P_{-}$, which corresponds to the electromechanical response from the poled states, ${ }^{19}$ and compare the $d_{31}^{\text {eff }}$ coefficient extracted from the slope $\kappa / V$ for each polarization direction. The effective $d_{31}^{\text {eff }}$ coefficients extracted using eqn (3) are $27.38 \pm 0.08 \mathrm{pm} \mathrm{V}^{-1}$
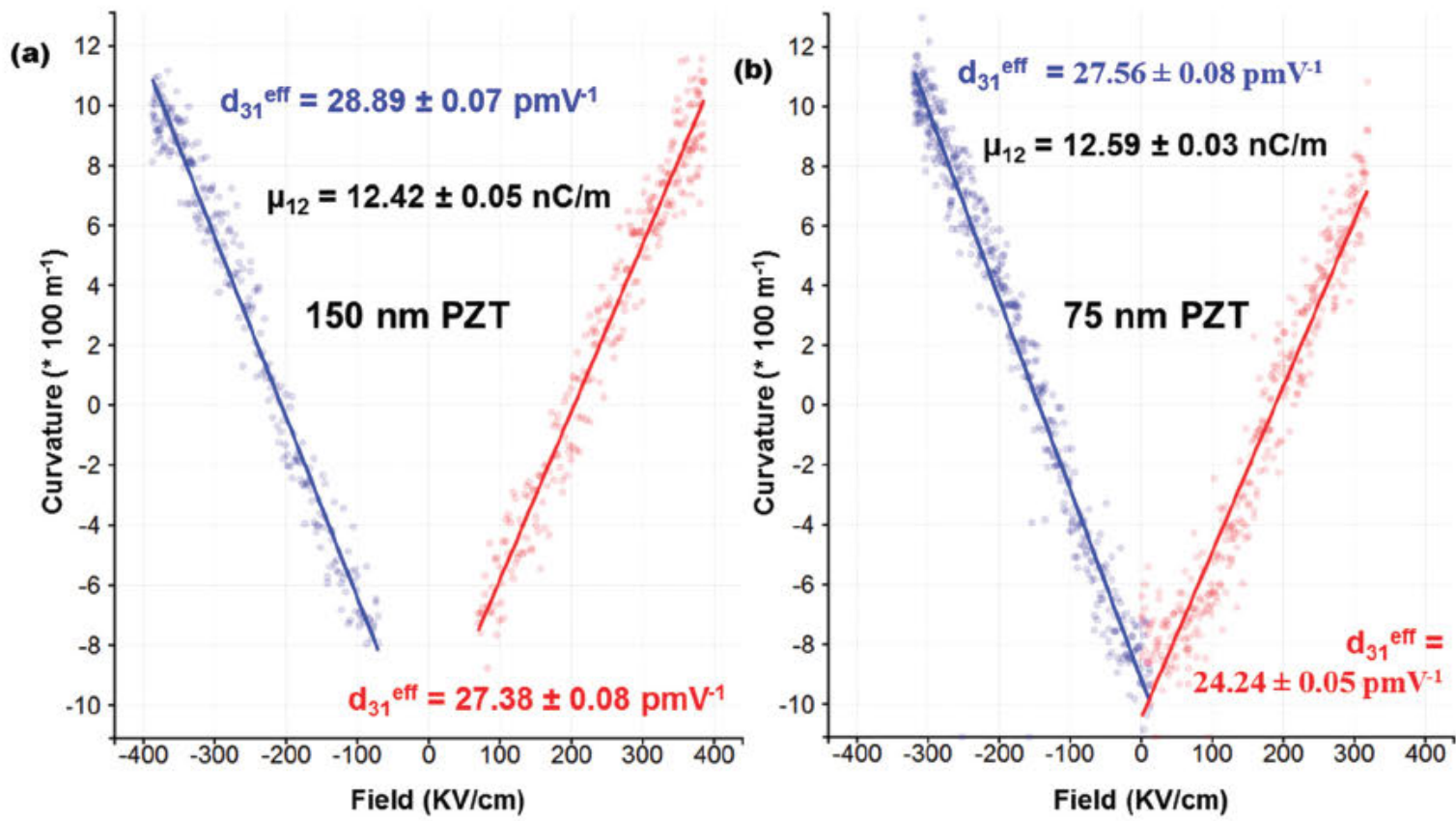

Fig. 4 (a) Data from reverse sweeps (i.e., poled state) of curvature $k$ as a function of the applied field for the $150 \mathrm{~nm}$ and (b) $75 \mathrm{~nm}$ thick PZT bimorph. The solid lines are least squares fits, the slope of which is the effective piezoelectric coefficient $d_{31}^{\text {eff }}$ (eqn (3)), from which the flexoelectric coefficient $\mu_{12}$ is extracted (eqn (4)). 

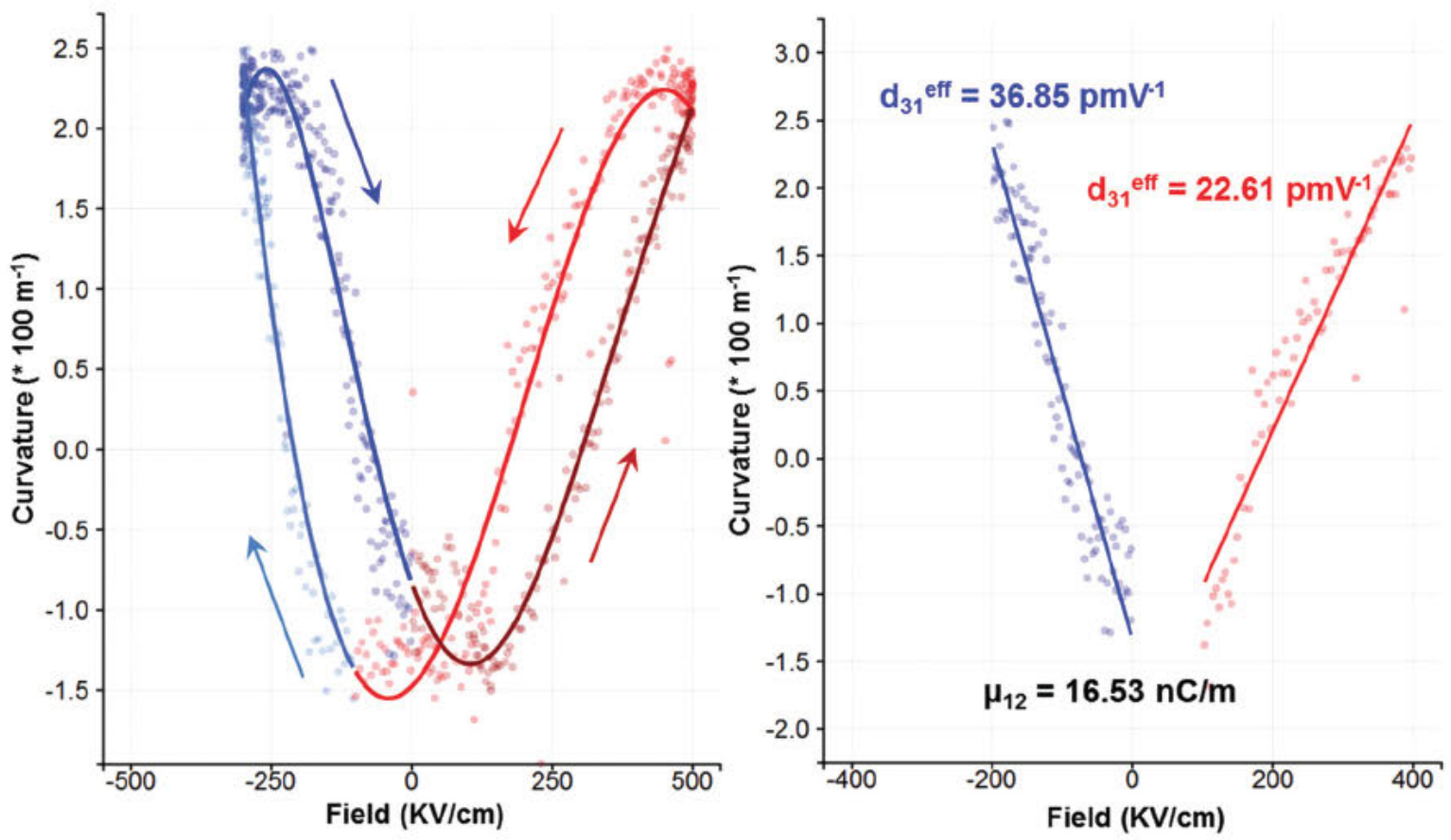

Fig. 5 (a) Butterfly hysteresis loops of curvature as a function of electric field for the $100 \mathrm{~nm}$ thick and PZT bimorph. In this case, a thick top elec trode of SRO is used to partially compensate the non centrosymmetric induced by the YSZ layer. The resulting structure results in larger asymmetry between piezoelectric coefficients. (b) Data from reverse sweeps (i.e., poled state) of curvature $k$ as a function of the applied field.

and $28.89 \pm 0.07 \mathrm{pm} \mathrm{V}^{-1}$ for $P_{-}$and $P_{+}$respectively, which is comparable to previous measurements on PZT cantilevers. ${ }^{23,24}$ The asymmetry in $d_{31}^{\text {eff }}$ is $5.36 \%$. Using eqn (4), we can use this asymmetry to calculate the flexoelectric coefficient, obtaining $\mu_{12}=12.42 \pm 0.05 \mathrm{nC} \mathrm{m}^{-1}$. Experimentally the flexoelectric coefficient of thin film PZT is an unknown and cannot be compared, but the measured values are in good agreement with theoretical predictions. ${ }^{4,25}$ We arrive at comparable values of $26.49 \mathrm{pm} \mathrm{V}^{-1}$ and $27.95 \mathrm{pm} \mathrm{V}^{-1}$ for the effective piezoelectric constants and a comparable value of $\mu_{12}=11.25 \pm 0.05 \mathrm{nC} \mathrm{m}^{-1}$ for the flexoelectric coefficient if we use a self-consistent continuum model for our cantilevers. ${ }^{8}$

In order to verify that the observed asymmetry in piezoelectric coefficients is due to flexoelectricity, we have conducted the same set of measurements on a PZT bimorph structure but with half the thickness for both the PZT film (75 nm), and the elastic layer $(75 \mathrm{~nm})$. The thickness of the top and bottom SRO electrodes is still $25 \mathrm{~nm}$. In Fig. 4(b), we plot the $\kappa-E$ for the $75 \mathrm{~nm}$ thick PZT bimorph. In this case, the measured $d_{31}^{\text {eff }}$ coefficients are $24.24 \pm 0.05 \mathrm{pm} \mathrm{V}^{-1}$ and $27.56 \pm 0.08 \mathrm{pm} \mathrm{V}^{-1}$ for $P_{-}$and $P_{+}$ respectively. The asymmetry is $12.81 \%$, over twice as much as for the twice-as-thick cantilever, as expected from eqn (4). The calculated flexoelectric coefficient in this case is $\mu_{12}=12.59 \pm 0.03 \mathrm{nC}$ $\mathrm{m}^{-1}$, almost identical to that of the thicker film, providing further evidence for the flexoelectric origin of the asymmetry.

It is now clear that flexoelectricity induces an asymmetry in the $\kappa-E$ curve of the PZT bimorph structure; however, to achieve a clear two state functionality, we need the bending moments generated by piezoelectricity and flexoelectricity to be of comparable magnitude. One way would be to further reduce the thickness of the flexoelectric layer, but maintaining the integrity of ultra-thin cantilevers is a difficult engineering challenge. Alternatively, we could reduce the thickness of the elastic clamping layer (YSZ), which simultaneously enhances the flexoelectric bending and reduces the piezoelectric one. Again, however, owing to technological issues the scaling of the elastic/template layer is not feasible, as it has a negative impact on the structural quality of the layers grown on top. But there is a third way. By depositing a thicker top electrode of SRO (100 nm), we can partially compensate the clamping asymmetry induced by the YSZ layer $(100 \mathrm{~nm})+$ SRO bottom electrode $(25 \mathrm{~nm})$ on the $100 \mathrm{~nm}$ thick PZT bimorph structure.

This moves the torque neutral axis $\left(z_{\mathrm{m}}\right)$ of the PZT bimorph closer to the center of the PZT thin film, resulting in comparable magnitudes of the bending moments arising from flexoelectricity and piezoelectricity. In Fig. 5, we plot the $\kappa-E$ for the aforementioned device. The measured $d_{31}^{\text {eff }}$ coefficients show a very large asymmetry $\sim 47 \%$ : $d_{31}^{\text {eff }}$ (piezo + flexo) $=36.85 \pm$ $0.29 \mathrm{pm} \mathrm{V}^{-1}$ and $d_{31}^{\text {eff }}($ piezo - flexo $)=22.61 \pm 0.21 \mathrm{pm} \mathrm{V}^{-1}$ for $P_{-}$and $P_{+}$respectively. The calculated flexoelectric coefficient in this case is $\mu_{12}=16.53 \pm 0.61 \mathrm{nC} \mathrm{m}^{-1}$, in good correspondence with the other ferroelectric bimorph cantilevers.

\section{Conclusions}

The collaboration $v s$. competition dynamics between flexoelectricity and ferroelectricity can be exploited not only to enhance 
the electromechanical performance of cantilevers, ${ }^{10}$ but also to design fundamentally new electromechanical devices. In particular, it allows creating an asymmetric piezoelectricity amplifier, whose effective piezoelectric coefficients are determined by the sign of the ferroelectric polarisation and which thus behave as electromechanical logic devices. Looking beyond PZT, lead free piezoelectrics like barium titanate $(\mathrm{BTO})^{26}$ display larger flexoelectric coefficients and smaller piezoelectric coefficients, and this broadens the range of geometrical parameters for which diode-like behaviour can in principle be achieved. In fact, BTO bimorph structures, operating around the Curie temperature, would allow tunability of the full palette of electromechanical functionalities and asymmetries on a single device.

\section{Author Contributions}

G.C. and U.B. conceived and designed the experiments; N.B. designed and made the cantilevers under the supervision of G.R.; U.B. performed the measurements and made the analytical model under the supervision of G.C.; A.A. performed the self-consistent continuum modelling and simulations; E.S. made the software for the analysis of the DHM images; U.B. and G.C. wrote the paper.

\section{Acknowledgements}

The work was funded by an ERC Starting Grant from the EU (Project No. 308023), a National Plan grant from Spain (FIS2013-48668-C2-1-P) and the Severo Ochoa Excellence programme. The authors are grateful to discussions with Etienne Cuche, Jerome Parent, and Yves Emery from lyncee tec Laussane.

\section{References}

1 S. H. Baek, J. Park, D. M. Kim, V. a. Aksyuk, R. R. Das, S. D. Bu, D. a. Felker, J. Lettieri, V. Vaithyanathan, S. S. N. Bharadwaja, N. Bassiri-Gharb, Y. B. Chen, H. P. Sun, C. M. Folkman, H. W. Jang, D. J. Kreft, S. K. Streiffer, R. Ramesh, X. Q. Pan, S. Trolier-McKinstry, D. G. Schlom, M. S. Rzchowski, R. H. Blick and C. B. Eom, Science, 2011, 334, 958-961.

2 P. Muralt, N. Ledermann, J. Paborowski, A. Barzegar, S. Gentil, B. Belgacem, S. Petitgrand, A. Bosseboeuf and N. Setter, IEEE Trans. Ultrason. Ferroelectr. Freq. Control, 2005, 52, 2276-2288.

3 A. K. Tagantsev and A. S. Yurkov, J. Appl. Phys., 2012, 112, 044103.

4 P. Zubko, G. Catalan and A. K. Tagantsev, Annu. Rev. Mater. Res., 2013, 43, 387-421.
5 T. D. Nguyen, S. Mao, Y. W. Yeh, P. K. Purohit and M. C. McAlpine, Adv. Mater., 2013, 25, 946-974.

6 E. Bursian and N. Trunov, Sov. Phys. Solid State, 1974, 16, 760-762.

7 E. Bursian and O. I. Zaikovskii, Sov. Phys. Solid State, 1968, 10, 1121.

8 A. Abdollahi, C. Peco, D. Millán, M. Arroyo and I. Arias, Computational Evaluation of the Flexoelectric Effect in Dielectric Solids, J. Appl. Phys., 2014, 116, 093502.

9 V. G. Zalesskii and E. D. Rumyantseva, Phys. Solid State, 2014, 56, 1352-1354.

10 M. Majdoub, P. Sharma and T. Çă̆in, Phys. Rev. B: Condens. Matter, 2008, 78, 121407.

11 A. Abdollahi and I. Arias, J. Appl. Mech., 2015, 82, 121003.

12 P. Hana, M. Marvan, L. Burianova, S. J. Zhang, E. Furman and T. R. Shrout, Ferroelectrics, 2006, 336, 137-144.

13 N. Banerjee, G. Koster and G. Rijnders, Appl. Phys. Lett., 2013, 142909, 0-5.

14 M. D. Nguyen, R. J. A. Steenwelle, J. M. Dekkers, D. H. A. Blank and G. Rijnders, Nano/Micro Eng. Mol. Syst. 2009. NEMS 2009. 4th IEEE Int. Conf., 2009, pp. 649-652.

15 M. Dekkers, M. D. Nguyen, R. Steenwelle, P. M. Riele, D. H. A. Blank and G. Rijnders, Appl. Phys. Lett., 2012, 95, 012902.

16 N. Banerjee, E. P. Houwman, G. Koster and G. Rijnders, Appl. Mater., 2014, 2, 096103.

17 E. Cuche, Y. Emery and F. Montfort, Nat. Photonics, 2009, 3, 633-635.

18 Y. Cotte, F. Toy, P. Jourdain and N. Pavillon, Nat. Photonics, 2013, 7, 113-117.

19 D. Damjanovic, in The Science of Hysteresis, 2006, vol. 3, pp. 337-465.

20 P. Zubko, G. Catalan, a. Buckley, P. Welche and J. Scott, Phys. Rev. Lett., 2007, 99, 167601.

21 S. H. Crandall, T. J. Lardner and N. C. Dahl, An introduction to the mechanics of solids: with SI units, 1999, vol. 3 .

22 M. S. Weinberg, J. Microelectromech. Syst., 1999, 8, 529533.

23 M. Dekkers, H. Boschker, M. van Zalk, M. Nguyen, H. Nazeer, E. Houwman and G. Rijnders, J. Micromech. Microeng., 2013, 23, 025008.

24 H. Nazeer, M. D. Nguyen, Ö. S. Sukas, G. Rijnders, L. Abelmann and M. C. Elwenspoek, J. Microelectromech. Syst., 2014, 24, 166-173.

25 G. Catalan, A. Lubk, A. H. G. Vlooswijk, E. Snoeck, C. Magen, A. Janssens, G. Rispens, G. Rijnders, D. H. A. Blank and B. Noheda, Nat. Mater., 2011, 10, 963967.

26 J. Narvaez, S. Saremi, J. Hong, M. Stengel and G. Catalan, Phys. Rev. Lett., 2015, 115, 1-5. 